\title{
DIE MITARBEITER DES BANDES
}

Prof. Dr. Werner T. Angress, Dept. of History, State University of New York at Stony Brook, Stony Brook, N. Y. 11794, USA

Ingrid Anker, M. A., Wissenschaftliche Rätin, Karolingerstr. 26, 8031 Gilching

Dr. Heinz-Ludger Borgert, Archivrat z. A., Alemannenstr. 11, 7809 Denzlingen

Dr. Martin van Creveld, Dept. of History, Hebrew University of Jerusalem, Jerusalem

Dr. H. R. Delporte, Wiss. Direktor a. D., Sundgauallee 55, 7800 Freiburg i. Br.

Dr. Jost Dülffer, Privatdozent, Hist. Seminar der Universität Köln, Albertus-Magnus-Platz, 5000 Köln 41

Johannes Fischer, Oberst a. D., Sonnenwiese 10, 7803 Gundelfingen-Wildtal

Prof. Dr. Konrad Fuchs, Hist. Seminar der Universität Mainz, Saarstr. 21, 6500 Mainz

Albrecht Götz v. Olenhusen, Rechtsanwalt, Holbeinstr. 12, 7800 Freiburg i. Br.

Dr. Friedhelm Golücke, Wiss. Assistent an der Universität Paderborn, Giersstr. 22/24, 479 Paderborn

Dr. Peter Gosztony, Leiter der Stiftung Schweizerische Osteuropa-Bibliothek, Jubiläumsstr. 41, CH-3005 Bern, Schweiz

Dr. Waldis Greiselis, Oberstlt., Ottweiler Str. 15, 3000 Hannover 71

Dr. Horst Gründer, Wiss. Assistent, Hist. Seminar, Westfälische Wilhelms-Universität, Domplatz 20-22, 4400 Münster (Westf.)

Dr. Othmar Hackl, Oberst i. G., Amtschef des Militärgeschichtlichen Forschungsamtes

Dr. Jürgen Heideking, Wiss. Angest., Seminar für Zeitgeschichte, Universität Tübingen, Wilhelmstr.

36, 7400 Tübingen

Prof. Dr. Peter Hoffmann, Dept. of History, McGill University, 855 Sherbrooke Street West, Montreal PQ, H3A 2T7, Canada

Prof. Dr. Heinz Hürten, Kath. Universität Eichstätt, Ostenstr. 26-28, 8078 Eichstätt

Karl-Heinz Hummel, Oberstlt. a. D., Gewerbestr. 40, 7803 Gundelfingen (Breisgau)

Dr. Hans-Christoph Junge, Studienassessor, Zum Wittmoos 8, 7750 Konstanz 19

Dr. Manfred Kehrig, Oberstlt. i. G., Leiter des Bundesarchiv-Militärarchivs, Wiesentalstr. 10, 7800 Freiburg i. Br.

Prof. Dr. Manfred Knapp, Fachbereich Gesellschaftswissenschaften, Johann Wolfgang Goethe-Universität, Senckenberganlage 15, 6000 Frankfurt a. M.

Dr. Bernhard R. Kroener, Wiss. Angest., Militärgeschichtliches Forschungsamt

Dr. Gerd Krumeich, Wiss. Assistent, Hist. Seminar der Universität Düsseldorf, Universitätsstr. 1, 4000 Düsseldorf 1

Dr. Alfred Mechtersheimer, Brahmsstr. 10 a, 8011 Vaterstetten

Rolf-Dieter Müller, Wiss. Angest., Militärgeschichtliches Forschungsamt

Dr. Norbert Ohler, Akad. Oberrat, Im Dorf 46, 7801 Horben

Prof. Dr. Eckardt Opitz, Heimfelder Str. 76, 2100 Hamburg 90

Dr. Léon Papeleux, Rue Belle Jardinière 421, B-4900 Angleur, Belgien

Dr. Jens Petersen, Deutsches Hist. Inst., Via Aurelia Antica 391, I-00165 Roma

Dr. Reiner Pommerin, Wiss. Assistent, Hist. Seminar der Universität Köln, Albertus-Magnus-Platz, 5000 Köln 41, z. Z. Harvard University, 5 Bryant Street, Cambridge, Mass. 02138, USA

Dr. Klaus-Jörg Ruhl, Keltenring 60, 7815 Kirchzarten

Helmut Schiller, Oberstlt. a. D., Vogelsang 17, 7814 Niederrimsingen b. Breisach am Rhein

Thomas Schnabel, Vogesenstr. 4, 7803 Gundelfingen (Breisgau) 
Reinhard Starck, Wörrstädter Str. 76, 6505 Nierstein 1

em. Prof. Dr. jur. Hans Thieme, Rehhagweg 19, 7800 Freiburg i. Br.

Dr. Gerd R. Ueberschär, Wiss. Rat, Militärgeschichtliches Forschungsamt

Dr. Hans Umbreit, Wiss. Oberrat, Militärgeschichtliches Forschungsamt

Dr. Bernhard Unckel, Oberstudienrat, Schückingstr. 15, 3550 Marburg a. d. Lahn

Dr. Bernd Wegner, Wiss. Angest., Militärgeschichtliches Forschungsamt

Hans-Heinrich Wilhelm, Bonifaziusstr. 19, 1000 Berlin 27

Prof. Dr. Gerhard Wirth, Seminar für Alte Geschichte der Universität Bonn, Am Hof 1 e, 5300 Bonn 